\title{
Idiopathic Right Ventricular Arrhythmias With Changes in the QRS Morphology After Ablation
}

\author{
Mohamad El Mohebํaㅅ Marwan Refaat ${ }^{2}$ \\ ${ }^{1}$ Massachusetts General Hospital \\ ${ }^{2}$ American University of Beirut Medical Center
}

July 7, 2020

Idiopathic ventricular arrhythmias (VA) is defined as premature ventricular complexes (PVCs) or ventricular tachycardias (VT) that occur in the absence of structural heart disease. Endocardial radiofrequency (RF) ablation is often curative for idiopathic VA. The success of the procedure depends on the ability to localize the abnormal foci accurately. These arrhythmias typical originate from the right ventricular outflow tract (RVOT), specifically from the superior septal aspect, but can also originate from the left ventricular outflow tract (LVOT) and the coronary cusps. ${ }^{1}$ The QRS electrocardiogram (ECG) characteristics have been helpful in patients with VAs, patient with accessory pathways and patients who have pacemakers. ${ }^{2}$ VAs originating from the RVOT have typical ECG findings with a left bundle branch block (LBBB) morphology and an inferior axis. ${ }^{3}$

In the current issue of the Journal of Cardiovascular Electrophysiology, Hisazaki et al. describe five patients with idiopathic VA suggestive of RVOT origin and who required ablation in the left-sided outflow tract (OT) in addition to the initial ablation in the RVOT for cure to be achieved. Patients exhibited monomorphic, LBBB QRS pattern with an inferior axis on ECG, consistent with the morphology of VAs originating from the RVOT. Interestingly, all patients had a common distinct ECG pattern: qs or rs (r [?] $5 \mathrm{~mm}$ ) pattern in lead I, Q wave ratio[aVL/aVR] $>1$, and dominant $\mathrm{S}$-waves in leads $\mathrm{V} 1$ and $\mathrm{V} 2$. Mapping of the right ventricle demonstrated early local activation time during the VA in the posterior portion of the RVOT, matching the QRS morphology obtained during pacemapping. Despite RF energy delivery to the RV, the VAs recurred shortly after ablation in four patients and had no effect at all in one patient. A change in the QRS morphology was noted on the ECG that had never been observed before the procedure. The new patterns were suggestive of left-sided OT origin: the second VAs exhibited an increase in the Q wave ratio $[\mathrm{aVL} / \mathrm{aVR}]$ and $\mathrm{R}$ wave amplitude in lead $\mathrm{V} 1$, decrease in the $\mathrm{S}$ wave amplitude in lead V1, and a counterclockwise rotation of the precordial R-wave transition. Early activation of the second VA could not be found in the RVOT, and the earliest activation time after mapping the LV was found to be relatively late. Real-time intracardiac echocardiography and 3D mapping systems were used to determine the location immediately contralateral to the initial ablation site in the RVOT. Energy was then delivered to that site which successfully eliminated the second VA. The authors postulated that the second VAs shared the same origins as the first VAs, and the change in QRS morphology is likely attributed to a change in the exit point or in the pathway from the origin to the exit point. The authors further explained that the VAs originated from an intramural area of the superior basal LV surrounded by the RVOT, LVOT and the transitional zone from the great cardiac vein to the anterior interventricular vein (GCV-AIV).

A limitation of this study is that GCV-AIV ablation was not attempted; however, the authors' approach is safer and was successful in eliminating VA. Another limitation is that left-sided OT mapping was not initially performed. Nevertheless, given the ECG characteristics, local activation time, and mapping, it was appropriate to attempt a RVOT site ablation. 
Overall, the authors should be commended for their effort to describe in detail patients with idiopathic VAs that required ablation in the left-sided OT following ablation in the RVOT. Although change in QRS morphology after ablation has been previously described, the authors were the first to describe the ECG patterns of these patients. ${ }^{4-7}$ The results of this study have important clinical implications. First, the authors have demonstrated the importance of anatomical approach from the left-sided OT for cure to be achieved. Second, insight into the location of the origin of the VA may be helpful to physicians managing patients with VAs from the RVOT. Finally, continuous monitoring of the ECG during ablation for a change in QRS morphology should be considered to identify patients who will require further ablation. We have summarized in Table 1 important ECG characteristics indicative VA of specific origins, based on the findings of this study and previous studies in the literature. ${ }^{3,8-15}$

\section{REFERENCES}

1. Morady F, Kadish AH, DiCarlo L, et al. Long-term results of catheter ablation of idiopathic right ventricular tachycardia. Circulation 1990;82(6):2093-9.

2. Refaat M, Mansour M, Singh JP, Ruskin JN, Heist EK. Electrocardiographic Characteristics in Right Ventricular Versus Biventricular Pacing in Patients With Paced Right Bundle Branch Block QRS Pattern. J Electrocardiol Mar-Apr 2011; 44 (2): 289-95.

3. Josephson ME, Callans DJ. Using the twelve-lead electrocardiogram to localize the site of origin of ventricular tachycardia. Hear Rhythm 2005;2(4):443-6.

4. Tada H, Hiratsuji T, Naito S, et al. Prevalence and characteristics of idiopathic outflow tract tachycardia with QRS alteration following catheter ablation requiring additional radiofrequency ablation at a different point in the outflow tract. Pacing Clin Electrophysiol 2004;27(9):1240-9.

5. Shirai Y, Liang JJ, Garcia FC, et al. QRS morphology shift following catheter ablation of idiopathic outflow tract ventricular arrhythmias: prevalence, mapping features, and ablation outcomes. J Cardiovasc Electrophysiol 2018;29(12):1664-71.

6. Pavlović N, Knecht S, Kühne M, Sticherling C. Changing exits in ventricular outflow tract tachycardia. Hear Rhythm 2014;11(8):1495-6.

7. Lokhandwala YY, Vora AM, Naik AM, Nabar A, Kavthale S. Dual morphology of idiopathic ventricular tachycardia. J Cardiovasc Electrophysiol 1999;10(10):1326-34.

8. Ouyang F, Fotuhi P, Ho SY, et al. Repetitive monomorphic ventricular tachycardia originating from the aortic sinus cusp: electrocardiographic characterization for guiding catheter ablation. J Am Coll Cardiol 2002;39(3):500-8.

9. Xie S, Kubala M, Liang JJ, et al. Lead IR-wave amplitude to differentiate idiopathic ventricular arrhythmias with left bundle branch block right inferior axis originating from the left versus right ventricular outflow tract. J Cardiovasc Electrophysiol 2018;29(11):1515-22.

10. Berruezo A, Mont L, Nava S, Chueca E, Bartholomay E, Brugada J. Electrocardiographic recognition of the epicardial origin of ventricular tachycardias. Circulation 2004;109(15):1842-7.

11. Yoshida N, Yamada T, Mcelderry HT, et al. A novel electrocardiographic criterion for differentiating a left from right ventricular outflow tract tachycardia origin: the V2S/V3R index. J Cardiovasc Electrophysiol 2014;25(7):747-53.

12. PARK K, KIM Y, Marchlinski FE. Using the surface electrocardiogram to localize the origin of idiopathic ventricular tachycardia. Pacing Clin Electrophysiol 2012;35(12):1516-27.

13. Good E, Desjardins B, Jongnarangsin K, et al. Ventricular arrhythmias originating from a papillary muscle in patients without prior infarction: A comparison with fascicular arrhythmias. Hear Rhythm [Internet] 2008;5(11):1530-7. Available from: http://www.sciencedirect.com/science/article/pii/S154752710800862X 
14. Doppalapudi H, Yamada T, McElderry HT, Plumb VJ, Epstein AE, Kay GN. Ventricular tachycardia originating from the posterior papillary muscle in the left ventricle: a distinct clinical syndrome. Circ Arrhythmia Electrophysiol 2008;1(1):23-9.

15. Sekiguchi Y, Aonuma K, Takahashi A, et al. Electrocardiographic and electrophysiologic characteristics of ventricular tachycardia originating within the pulmonary artery. J Am Coll Cardiol 2005;45(6):887-95.

\section{Hosted file}

Table.docx available at https://authorea.com/users/317502/articles/467314-idiopathic-rightventricular-arrhythmias-with-changes-in-the-qrs-morphology-after-ablation 